\title{
Radial velocities and iron abundances of field RR Lyraes. II.
}

\author{
E. Solano ${ }^{1}$, R. Garrido ${ }^{2}$, J. Fernley ${ }^{3}$, and T.G. Barnes ${ }^{4}$ \\ 1 INSA; ESA IUE Observatory, P.O. Box 50727, 28080 Madrid, Spain \\ 2 Instituto de Astrofísica de Andalucía, CSIC, P.O. Box 3004, 18080, Granada, Spain \\ ${ }^{3}$ IUE Observatory, P.O. Box 50727, 28080 Madrid, Spain \\ 4 McDonald Observatory, University of Texas, Austin, Texas 78712, U.S.A.
}

Received October 29; accepted December 5, 1996

\begin{abstract}
This is the second of the papers devoted to derive radial velocities and iron abundances of field $\mathrm{RR}$ Lyraes observed by HIPPARCOS. Our abundances show good agreement with those in the literature obtained both from photometric ( $\Delta S$ index) and spectroscopic methods. Binary candidates and stars misclassified as RR Lyraes in the original HIPPARCOS list have been also identified. ${ }^{1}$
\end{abstract}

Key words: stars: variables — stars: abundances stars: kinematics

\section{Introduction}

In this paper we present the radial velocities and iron abundances for field RR Lyraes observed by the HIPPARCOS satellite. By combining these data with the proper motions and $V$ magnitudes from HIPPARCOS it will be possible to determine the RR Lyrae absolute magnitude-metallicity relation using the method of Statistical Parallax (e.g. Hawley et al. 1986; Strugnell et al. 1986). In an earlier paper (Fernley \& Barnes 1997, hereafter Paper I), systemic velocities and iron abundances were presented for $56 \mathrm{RR}$ Lyraes using echelle spectra taken at McDonald Observatory, Texas. In this paper we present similar data for a further 45 RR Lyraes observed at either Calar Alto Observatory, Spain or Sutherland Observatory, S. Africa.

\section{Observations and data reduction}

The observations were performed in different observing runs carried out in 1994-1995 at two observatories: the

Send offprint requests to: E. Solano; esm@vilspa.esa.es

1 Appendix is only available in electronic form at the CDS via anonymous ftp to cdsarc.u-strasbg.fr (130.79.128.5) or via http://cdsweb.u- strasbg.fr/Abstract.html
Observatorio de Calar Alto (Almería, Spain) and the Sutherland Observatory (South Africa). Observations at Calar Alto were made in May and December 1994 using the Coudé spectrograph on the $2.2 \mathrm{~m}$ telescope. The wavelength covers $\approx 230 \AA(4160 \AA-4390 \AA)$. The resolving power was 22000 which corresponds to a reciprocal spectral dispersion of $0.20 \AA /$ pixel at $\mathrm{H} \gamma$. Observations at Sutherland were made in July 1995 using the Image Tube Spectrograph and the RPCS detector on the $1.9 \mathrm{~m}$ telescope. The spectra cover the wavelength range $4070 \AA-4490 \AA$. The resolving power was 19000 which corresponds to a reciprocal spectral dispersion of $0.23 \AA /$ pixel.

The exposure times in all the runs were limited to 15 minutes for the RR Lyraes in order to prevent "phase blurring". In general, three spectra were obtained for each RR Lyrae. The first spectrum was arbitrarily assigned as phase zero and then, using the published periods from the GCVS, two further spectra were obtained separated by plus or minus a third of a cycle from the first spectrum.

The spectra were reduced using the FIGARO reduction package: the raw spectra were bias-subtracted, flatfield-corrected (two or more master flatfield exposure were taken every night) and background-subtracted before the spectral extraction. The wavelength calibration of the stellar spectra was done with thorium-argon comparison spectra. Because of the variations in the velocity zero point of the Image Tube Spectrograph at Sutherland (probably due to a mechanical slippage of the grating mount), arcs were taken before and after every observation. In the Calar Alto runs, arcs were taken at the beginning and the end of every night. Polynomial calibrations of wavelength as a function of pixel number were calculated for each comparison spectrum. A fourth order polynomial fit was used for Sutherland spectra whereas a second order polynomial fit was found adequate for the Calar Alto sample. In both cases a precision better than $0.04 \AA$ was achieved which corresponds to $\approx 2.8 \mathrm{~km} \mathrm{~s}^{-1}$ at $4200 \AA$. The continuum 
windows were chosen with the aid of the Atlas of Procyon (Griffin \& Griffin 1979) and the continuum level calculated by fitting a second-degree polynomial. The normalized spectra were then derived by dividing the extracted spectra by this continuum level. The signal-to-noise ratio of the spectra varied between 5 and 25 depending on the magnitude of the star and the quality of the seeing. In addition, several IAU radial velocity standard stars were observed each night to measure the zero-point offset in radial velocity of our measurements.

\section{Radial velocities}

A cross-correlation technique was used to calculate the radial velocities of the observed RR Lyrae. As a first step, the original wavelength range was logarithmically rebinned from 4189.4 to $4389.4 \AA$ for Calar Alto spectra and from 4073.6 to $4446.7 \AA$ for Sutherland spectra to 1024 and 2048 data points respectively. After this, the spectra were cross-correlated (using a Fast Fourier Transform technique) against the observed IAU radial velocity standards (Table 1). A 5\% cosine-bell was used to remove the start and end spectrum discontinuities. The cross-correlation peak was then fitted to a parabola, the maximum value of the parabola being the radial velocity difference between the standard and the target star. Finally, the heliocentric correction was applied using the standard IRAF package.

The phases of our observations were calculated using the published periods from the GCVS and an arbitrary zero-point. Systemic velocities were then determined by fitting these phased radial velocity measurements for each star to the "standard" RRab Lyrae radial velocity curve given by Liu (1991). A free parameter in the Liu curve is the amplitude. Liu found 22 RRab Lyraes with published radial velocity curves and these have a mean amplitude of $61.5 \mathrm{~km} \mathrm{~s}^{-1}$ with an rms scatter of $8.4 \mathrm{~km} \mathrm{~s}^{-1}$. Liu shows that there is a correlation between light curve amplitude and velocity amplitude; however, since the light curve amplitude is not known for many of the stars in our sample, we have used the mean amplitude of $61.5 \mathrm{~km} \mathrm{~s}^{-1}$ to construct the "standard" curve. Amongst the stars for which we have three well-phased spectra with good S/N there are 4 RRab Lyraes and 2 RRc Lyraes with complete radial velocity curves available in the literature. Comparing our values of the systemic velocity with the literature values for the 4 RRab Lyraes showed a mean difference of $1.7 \mathrm{~km} \mathrm{~s}^{-1}$ and an rms difference of $3.1 \mathrm{~km} \mathrm{~s}^{-1}$. For the RRc Lyraes, where the velocity amplitude is smaller and the velocity curve more symmetric, we determined the systemic velocity by simply taking a mean of our three measurements. Again, comparing our systemic velocities with the literature values showed, for the two RRc Lyraes, a mean difference of $1.5 \mathrm{~km} \mathrm{~s}^{-1}$ and an rms difference of $2.1 \mathrm{~km} \mathrm{~s}^{-1}$
Thus for those stars where we have three well-phased radial velocities and good $\mathrm{S} / \mathrm{N}$ spectra, a realistic $1-\sigma$ error in the systemic velocity is $\pm 3 \mathrm{~km} \mathrm{~s}^{-1}$. For those stars with fewer measurements and/or lower $\mathrm{S} / \mathrm{N}$ the errors are larger. The full list of RR Lyraes, their systemic velocities and associated errors is given in the Appendix (available electronically).

Table 1. IAU radial velocity standards

\begin{tabular}{lr}
\hline Identification & $\begin{array}{r}\text { Velocity } \\
\left(\mathrm{km} \mathrm{s}^{-1}\right)\end{array}$ \\
\hline HR 0033 & 14.7 \\
HR 1101 & 27.9 \\
HD 65934 & 35.0 \\
HR 4540 & 5.0 \\
HR 5694 & 53.5 \\
HR 7560 & 0.1 \\
HR 8969 & 5.3 \\
\hline
\end{tabular}

\subsection{Binary candidates}

Because the Horizontal Branch is a relatively short-lived phase of stellar evolution, most companion stars will be much fainter than the RR Lyrae itself, i.e. the companions will either be low-mass main sequence stars or white dwarfs. In general, therefore, the only methods available to detect companions are to look for variations in either the time of maximum light or the systemic velocity. In Table 2 we list those RR Lyraes which show significant differences between our values and previous values of the systemic velocity. Examination of the spectra of these stars showed no evidence of line doubling for any of them.

The only one of stars included in Table 2 previous suspected to be a member of a binary is TU UMa. Saha \& White (1990), using published times of maximum light, calculated the orbital parameters for TU UMa and our value of the systemic velocity is not inconsistent with their prediction. For the other stars, a literature search showed there were insufficient published times of maximum light to attempt an orbital solution and clearly it would be of value to place these stars in a long-term observing programme in order to acquire these data.

\section{Effective temperatures and metallic abundances}

It is well established that the Balmer lines are good temperature indicators for $T_{\text {eff }} \leq 8500 \mathrm{~K}$ because of their small gravity and metallicity dependence (Smalley \& Dworetsky 1993; Furhmann et al. 1994). In this work, effective temperatures for both non-variable and RR Lyrae stars have been obtained from the comparison, using a least-squares fitting technique, between the observed $\mathrm{H} \gamma$ 
Table 2. Binary candidates

\begin{tabular}{lccl}
\hline Identification & \multicolumn{2}{c}{$\begin{array}{c}\text { Systemic velocity } \\
\text { Phis work } \\
\left(\mathrm{km} \mathrm{s}^{-1}\right)\end{array}$} & $\begin{array}{c}\text { Refious work } \\
\left(\mathrm{km} \mathrm{s}^{-1}\right)\end{array}$ \\
\hline TY Aps & $90 \pm 3$ & $129 \pm 14,60 \pm 16$ & 9,17 \\
BX Dra & $-24 \pm 3$ & $75 \pm 30$ & 9 \\
XX Hya & $32 \pm 30$ & $52 \pm 24,-10 \pm 35,95 \pm 10$ & $9,14,16$ \\
BX Leo & $-7 \pm 15$ & $27 \pm 5$ & 16 \\
CN Lyr & $13 \pm 20$ & $67 \pm 30,27 \pm 5$ & 9,16 \\
TU UMa & $96 \pm 3$ & $84 \pm 2,90 \pm 2$ & 1,8 \\
& & $77 \pm 2,101 \pm 5$ & 12,16 \\
\hline
\end{tabular}

Table 3. Effective temperatures, surface gravities and $[\mathrm{Fe} / \mathrm{H}]$ abundances of the non-variable stars. The number in parenthesis indicates the number of lines used in the abundance determination

\begin{tabular}{|c|c|c|c|c|c|}
\hline Identification & Eff. Temp (K) & Previous work & {$[\mathrm{Fe} / \mathrm{H}]$} & Previous work & $\log g$ \\
\hline HR 0033 & 6279 & $6204^{1} / 6156^{7}$ & $-0.12(8)$ & $-0.38^{1} /-0.40^{7}$ & $4.07^{1} / 4.12^{7}$ \\
\hline HR 0033 & 6254 & & $-0.21(9)$ & & \\
\hline HR 0033 & 6346 & & $-0.27(7)$ & & \\
\hline HR 2943 & 6542 & $6500-6750^{12}$ & $-0.07(5)$ & $0.00^{12}$ & $4.00^{12}$ \\
\hline HR 4540 & 6062 & $6095^{3} / 6120^{2} / 6176^{1}$ & $+0.08(5)$ & $0.13^{2} / 0.13^{1}$ & $4.22^{2} / 4.14^{1}$ \\
\hline HR 4540 & 6060 & & $+0.16(5)$ & & \\
\hline HR 4540 & 5973 & & $-0.04(6)$ & & \\
\hline HR 4540 & 6095 & & $+0.09(5)$ & & \\
\hline HR 5694 & 6204 & $6142^{6} / 6055^{8}$ & $+0.05(10)$ & $-0.12^{8}$ & \\
\hline HR 5694 & 6278 & & $-0.11(8)$ & & \\
\hline HR 5694 & 6102 & & $+0.02(7)$ & & \\
\hline HR 5694 & 6113 & & $+0.04(7)$ & & \\
\hline HR 5694 & 6258 & & $+0.06(6)$ & & \\
\hline HR 5694 & 6140 & & $+0.00(6)$ & & \\
\hline HR 5694 & 5950 & & $-0.16(6)$ & & \\
\hline HR 7560 & 6273 & $6146^{1} / 6360^{4}$ & $+0.00(7)$ & $0.09^{1} / 0.18^{5}$ & $4.14^{1} / 4.40^{5}$ \\
\hline HR 7560 & 6231 & & $+0.15(7)$ & & \\
\hline HR 7560 & 6190 & & $-0.10(5)$ & & \\
\hline HR 7560 & 6188 & & $+0.19(6)$ & & \\
\hline HR 7560 & 6132 & & $+0.13(6)$ & & \\
\hline HR 7560 & 6060 & & $+0.08(6)$ & & \\
\hline HR 8969 & 6318 & $6255^{1} / 6157^{7} / 6105^{9}$ & $-0.19(9)$ & $-0.17^{1} /-0.21^{7}$ & $4.16^{1} / 4.17^{9}$ \\
\hline HR 8969 & 6205 & & $-0.06(8)$ & & \\
\hline HR 8969 & 6184 & & $-0.15(5)$ & & \\
\hline HR 8969 & 5991 & & $-0.19(6)$ & & \\
\hline HR 8969 & 6021 & & $-0.15(6)$ & & \\
\hline HR 8969 & 6258 & & $-0.11(6)$ & & \\
\hline SAO 37362 & 6133 & $6132^{3} / 6212^{10} / 6205^{11}$ & $+0.07(3)$ & $0.0^{3} / 0.09^{10} /-0.16^{11}$ & $4.0^{3,12} / 4.17^{10}$ \\
\hline SAO 101826 & 6306 & $6220^{2} / 6233^{3} / 6333^{1}$ & $-0.23(6)$ & $-0.32^{2} /-0.16^{1}$ & $4.24^{2} / 4.25^{3}$ \\
\hline
\end{tabular}

1: Edvardsson et al. (1993); 2: Bell et al. (1994); 3: Alonso et al. (1996); 4: Sokolov (1995); 5: Borges et al. (1995); 6: Cayrel de Strobel et al. (1992); 7: King \& Boesgaard (1995); 8: Carney et al. (1987); 9: Favata et al. (1996); 10: Tomkin et al. (1995); 11: Blackwell et al. (1994); 12: Drake \& Laming (1995). 
Table 4. Adopted parameters and derived quantities for the RR Lyrae stars observed at Calar Alto. The number in parenthesis indicates the number of lines used in the abundance calculation

\begin{tabular}{|c|c|c|c|c|}
\hline Identification & Ephemeris & Phase & $\begin{array}{l}T_{\text {eff }} \\
(\mathrm{K})\end{array}$ & {$[\mathrm{Fe} / \mathrm{H}]$} \\
\hline AT And & $42343.420+0.616915^{2}$ & 0.09 & 6690 & $-1.14(1)$ \\
\hline SW And & $47116.185+0.442266^{3}$ & 0.86 & 5921 & $-0.36(6)$ \\
\hline $\mathrm{AE}$ Boo & $30388.203+0.314892^{2}$ & 0.20 & 6616 & $-0.95(4)$ \\
\hline AE Boo & & 0.59 & 6296 & $-1.18(3)$ \\
\hline AE Boo & & 0.28 & 6978 & $-0.99(3)$ \\
\hline AE Boo & & 0.94 & 6796 & $-0.96(3)$ \\
\hline RS Boo & $46948.720+0.377337^{1}$ & 0.47 & 6233 & $-0.31(4)$ \\
\hline RS Boo & & 0.87 & 6210 & $-0.34(4)$ \\
\hline RR Cet & $46773.724+0.553038^{1}$ & 0.65 & 6000 & $-1.24(2)$ \\
\hline SU Dra & $46833.664+0.66042^{1}$ & 0.67 & 5721 & $-1.97(5)$ \\
\hline SU Dra & & 0.99 & 7200 & $-1.42(2)$ \\
\hline SW Dra & $46495.754+0.569669^{1}$ & 0.30 & 6190 & $-0.70(4)$ \\
\hline SW Dra & & 0.00 & 7279 & $-0.84(2)$ \\
\hline XX Нyа & $39832.011+0.507741^{2}$ & 0.40 & 6839 & $-1.22(2)$ \\
\hline TT Lyn & $46773.679+0.597436^{1}$ & 0.74 & 5888 & $-1.27(2)$ \\
\hline TT Lyn & & 0.41 & 6223 & $-1.38(4)$ \\
\hline CN Lyr & $44486.334+0.411382^{2}$ & 0.09 & 6582 & $-0.09(2)$ \\
\hline CN Lyr & & 0.40 & 6173 & $-0.10(2)$ \\
\hline KX Lyr & $39630.870+0.440904^{2}$ & 0.20 & 6368 & $-0.30(1)$ \\
\hline KX Lyr & & 0.46 & 6248 & $-0.41(3)$ \\
\hline DH Peg & $44463.571+0.255510^{2}$ & 0.26 & 7285 & $-1.35(1)$ \\
\hline DZ Peg & $33891.308+0.607344^{2}$ & 0.41 & 6400 & $-1.21(3)$ \\
\hline VZ Peg & $38317.293+0.306486^{2}$ & 0.54 & 7010 & $-1.63(3)$ \\
\hline AR Per & $46773.473+0.425549^{1}$ & 0.24 & 6560 & $-0.23(2)$ \\
\hline AR Per & & 0.48 & 6143 & $-0.23(3)$ \\
\hline AP Ser & & & 6475 & $-1.62(3)$ \\
\hline VY Ser & $38466.244+0.714096^{1}$ & 0.24 & 6101 & $-1.46(2)$ \\
\hline VY Ser & & 0.62 & 5869 & $-1.42(2)$ \\
\hline T Sex & $46833.592+0.324712^{3}$ & 0.32 & 6650 & $-1.30(3)$ \\
\hline T Sex & & 0.62 & 7200 & $-1.27(2)$ \\
\hline T Sex & & 0.32 & 6737 & $-1.25(2)$ \\
\hline SX Uma & & & 6563 & $-1.75(1)$ \\
\hline SX Uma & & & 7342 & $-1.51(1)$ \\
\hline
\end{tabular}

1: Fernley \& Barnes (1996); 2: Kholopov et al. (1990); 3: Liu \& Janes (1990).

profile and a grid of synthetic profiles using ATLAS8 (Kurucz 1979). The gravity and metallicity were fixed to $\log g=2.75$ and $[M / H]=-1.0$ for the RR Lyrae stars and to $\log g=4.0$ and $[M / H]=0.0$ for the sample of non-variable stars. Effective temperatures of the observed stars are given in Tables 3,4 and 5 .

It has been pointed out that for stars cooler than $8500 \mathrm{~K}$, convection is starting to become efficient enough to modify the temperature gradient and hence the Balmer profiles with the exception of $\mathrm{H} \alpha$ (van't Veer-Menneret \& Megessier 1996). However, we have not found any evidence for this. Comparing our effective temperatures derived from $\mathrm{H} \gamma$ and those obtained from the literature for the non-variable stars shows a mean difference and a standard deviation of $-11 \pm 73 \mathrm{~K}$. Moreover, previous comparisons between effective temperatures derived from $\mathrm{H} \beta$ from the Infrared Flux method and spectrophotometric methods (Solano \& Fernley 1997) did not show systematic differences either.

The crowding of the observed spectral region made the line selection difficult and only a small number of Fe I lines were selected for the abundance calculation (Table 6): weak lines (with associated large errors due to the moderate $\mathrm{S} / \mathrm{N}$ ratio of our observations) and strong lines (sensitive to saturation effects) were not considered. The relatively low signal-to-noise together with the crowded spectral region also cause an uncertainty in the measured equivalent width. An estimation of this uncertainty was obtained by comparing the equivalent widths of lines of non-variable stars with two or more observations. This gave a typical error of $7 \%$. For the RR Lyrae stars, which 
Table 5. Same as Table 4 but for Sutherland observations

\begin{tabular}{lllll}
\hline Identification & Ephemeris & Phase & $\begin{array}{l}T_{\text {eff }} \\
(\mathrm{K})\end{array}$ & {$[\mathrm{Fe} / \mathrm{H}]$} \\
\hline TY Aps & $39726.275+0.501693^{1}$ & 0.16 & 7131 & $-0.66(5)$ \\
TY Aps & & 0.39 & 6194 & $-0.92(4)$ \\
XZ Aps & $28715.330+0.587434^{1}$ & 0.93 & 6579 & $-0.55(8)$ \\
BV Aqr & $37524.218+0.364048^{1}$ & 0.62 & 7011 & $-1.16(3)$ \\
BV Aqr & & 0.09 & 6868 & $-1.22(3)$ \\
BV Aqr & & 0.43 & 6394 & $-1.20(5)$ \\
BV Aqr & & 0.94 & 7138 & $-1.15(1)$ \\
RR Cet & $46773.724+0.553038^{3}$ & 0.77 & 6044 & $-1.29(5)$ \\
RR Cet & & 0.45 & 5979 & $-1.38(4)$ \\
RR Cet & & 0.26 & 6116 & $-1.29(6)$ \\
DX Del & $39367.340+0.472617^{1}$ & 0.21 & 6799 & $-0.31(5)$ \\
DX Del & & 0.48 & 6141 & $-0.38(8)$ \\
DX Del & & 0.59 & 6041 & $-0.41(6)$ \\
CS Eri & $38417.087+0.311331^{1}$ & 0.12 & 6928 & $-1.36(4)$ \\
CS Eri & & 0.31 & 6679 & $-1.45(4)$ \\
XZ Gru & $38260.438+0.34741^{1}$ & 0.68 & 6158 & $-1.37(6)$ \\
XZ Gru & & 0.03 & 6068 & $-1.12(5)$ \\
XZ Gru & & 0.43 & 6029 & $-1.36(7)$ \\
IK Hya & $38461.510+0.65^{1}$ & 0.25 & 6373 & $-1.24(1)$ \\
FW Lup & $42171.377+0.4841712^{1}$ & 0.80 & 6506 & $-0.11(7)$ \\
FW Lup & & 0.14 & 6405 & $-0.26(9)$ \\
FW Lup & & 0.36 & 6294 & $-0.14(7)$ \\
FW Lup & & 0.47 & 6164 & $-0.27(10)$ \\
VY Nor & $25535.249+0.375305^{1}$ & 0.63 & 6000 & $-2.01(1)$ \\
VW Scl & $27809.381+0.510915^{1}$ & 0.15 & 7121 & $-0.84(4)$ \\
VY Ser & $38466.244+0.714096$ & 0.82 & 6024 & $-1.30(6)$ \\
VY Ser & & 0.21 & 6335 & $-1.53(3)$ \\
VY Ser & & 0.54 & 5969 & $-1.65(2)$ \\
MT Tel & $42206.35+0.316897^{1}$ & 0.57 & 6832 & $-1.64(2)$ \\
MT Tel & & 0.92 & 7085 & $-1.62(2)$ \\
AM Vir & $26859.275+0.615089^{1}$ & 0.12 & 6493 & $-0.74(6)$ \\
AM Vir & & 0.20 & 6037 & $-1.11(5)$ \\
AM Vir & & 0.53 & 5932 & $-1.30(1)$ \\
UU Vir & $46878.822+0.475606^{2}$ & 0.31 & 6359 & $-0.70(7)$ \\
\hline & & & &
\end{tabular}

1: Kholopov et al. (1990); 2: Jones et al. (1988); 3: Fernley \& Barnes (1996).

generally have lower $\mathrm{S} / \mathrm{N}$, the error in $\mathrm{EW}$ is higher and we estimate $10 \%$.

Synthetic equivalent widths were calculated for a grid of temperatures and metallicities using the spectrum synthesis code XLINOP and the ATLAS8 models (Kurucz 1979). Log $g f$ values were taken from Thévenin (1989) (Table 6). The abundance values were calculated by computing the sum of squares of the differences between the observed equivalent widths and the synthetic equivalent widths for five values of metallicity. The sum of squares were then plotted against the metallicities and a parabola was fitted, the adopted metallicity being the minimum of the parabola. In order to check the internal consistency of the whole process we have calculated the abundances of Procyon (HR 2943) and some other standard stars. A microturbulence of $2.0 \mathrm{~km} \mathrm{~s}^{-1}$ and a surface gravity of $\log g=4.0$ were used for all the stars. The results are given in Table 3 . Comparing these values and those obtained from the literature shows good agreement (mean difference and a standard deviation of $0.03 \pm 0.09 \mathrm{dex}$ ).

The microturbulence velocity has been set to $\xi=3.6 \mathrm{~km} \mathrm{~s}^{-1}$ (Lambert et al. 1996) for most of the RR Lyrae stars since the scattered in the abundances derived from the lines was too large or the number of lines used too small to distinguish any systematic differences in the abundances derived from weak and strong lines. Also, following Fernley \& Barnes (1996), a value of $\log g=2.75$ was adopted for all the RR Lyrae stars.

The derived abundances from the individual RR Lyrae spectra are listed in Tables 4, 5. For stars with two or more abundance determinations it can be seen that there is good agreement, the differences are typically $\pm 0.1 \mathrm{dex}$. 
The final abundance for each RR Lyrae star is listed in the Appendix. Also listed are values from the literature. The two sets of values are compared in Fig. 1 where it can be seen that, with the exception of $\mathrm{XZ}$ Aps, there is reasonable agreement ( $r m s$ of the difference 0.18 dex). Since the literature values have a typical error of $\pm 0.13 \mathrm{dex}$, this rms difference implies a similar error in our work although there is some suggestion that our metallicities are systematically higher (mean difference 0.07 dex). The sensitivity of the derived abundances on the atmospheric parameters has also been studied. Errors of $\Delta T_{\text {eff }}=150 \mathrm{~K}$ and $\Delta \xi=0.3 \mathrm{~km} \mathrm{~s}^{-1}$ have been assumed for effective temperatures and microturbulence velocities which produce errors of $\Delta[\mathrm{Fe} / \mathrm{H}]=0.16$ dex and $\Delta[\mathrm{Fe} / \mathrm{H}]=0.12$ dex respectively. The influence of the errors in $\log g$ on the derived abundances is negligible.

From Tables 4, 5 we can see that stars with abundances calculated at phases out of the minimum light interval show good agreement with the values calculated at minimum light even if at these phases the stellar atmosphere may suffer from fast acceleration which produces large ranges in the physical parameters in a very short time-scale. Clementini et al. (1995) also found similar results. The explanation of this is related to the fact that the steepest variations in a $T_{\text {eff-phase diagram only occurs in }}$ the interval $0.9 \leq \phi \leq 0.0$ (Fernley et al. 1989). In the rest of the phase interval the variation of $T_{\text {eff }}$ is such that, if the ephemeris are accurate enough, the temperature can be determined with a small error. This is also valid for the variations in gravity (e.g. Liu \& Janes 1990) with the additional advantage of the little influence of $\log g$ on the abundance determination.

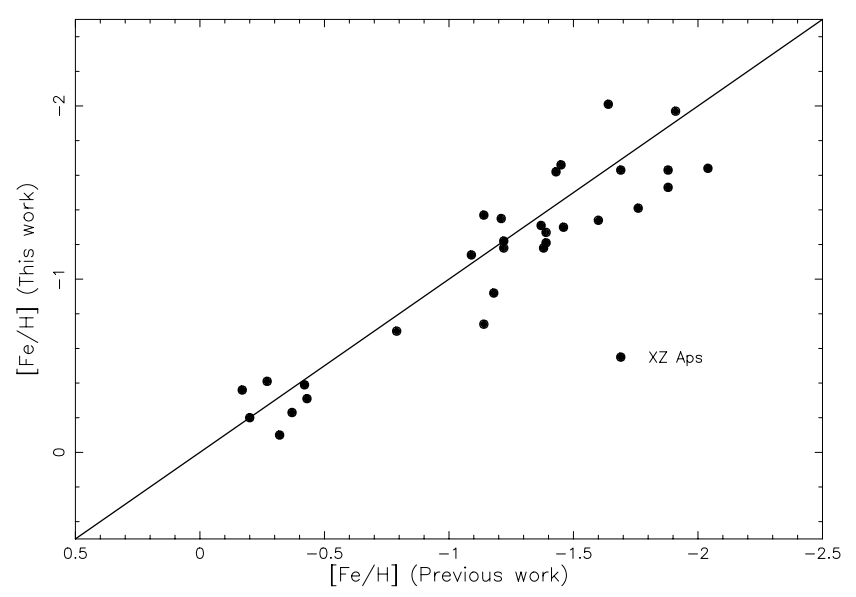

Fig. 1. A comparison between the $[\mathrm{Fe} / \mathrm{H}]$ values of the present work and previous work
Table 6. Adopted atomic parameters. Log $g f$ values and lower level energies were taken from Thévenin (1989) and Kurucz \& Peytremann (1975) respectively

\begin{tabular}{lcc}
\hline Identification & Log $g f$ & $\begin{array}{c}\text { Lower level energy } \\
\left(\mathrm{cm}^{-1}\right)\end{array}$ \\
\hline Fe I $\lambda 415.1945$ & -1.570 & 28604.605 \\
Fe I $\lambda 415.2056$ & -0.990 & 32873.617 \\
Fe I $\lambda 415.2169$ & -3.180 & 7728.056 \\
\hline Fe I $\lambda 415.3899$ & -0.590 & 27394.688 \\
Fe I $\lambda 415.4098$ & -1.500 & 27394.688 \\
Fe I $\lambda 415.4500$ & -1.040 & 22838.318 \\
Fe I $\lambda 415.4805$ & -0.640 & 27166.818 \\
\hline Fe I $\lambda 417.6577$ & -0.680 & 27166.818 \\
\hline Fe I $\lambda 418.7047$ & -0.660 & 19757.033 \\
Fe I $\lambda 418.7588$ & -1.320 & 27666.346 \\
Fe I $\lambda 418.7617$ & -2.410 & 29356.740 \\
Fe I $\lambda 418.7795$ & -0.650 & 19562.439 \\
\hline Fe I $\lambda 419.5329$ & -0.720 & 26874.548 \\
Fe I $\lambda 419.5618$ & -1.710 & 24335.760 \\
Fe I $\lambda 419.6208$ & -0.920 & 27394.688 \\
Fe I $\lambda 419.6533$ & -2.190 & 23783.613 \\
\hline Fe I $\lambda 421.3653$ & -1.550 & 22946.809 \\
\hline Fe I $\lambda 421.9355$ & -0.720 & 28819.945 \\
Fe I $\lambda 421.9419$ & -1.650 & 24118.814 \\
\hline Fe I $\lambda 422.2221$ & -0.980 & 19757.033 \\
\hline Fe I $\lambda 425.0130$ & -0.350 & 19912.494 \\
Fe I $\lambda 425.0787$ & -1.040 & 12560.930 \\
Fe I $\lambda 425.0893$ & -2.040 & 24772.018 \\
\hline Fe I $\lambda 440.4080$ & -2.530 & $31686.346 *$ \\
Fe I $\lambda 440.4750$ & -0.250 & $12560.930 *$ \\
\hline & & \\
\hline
\end{tabular}

* Only used in metal-poor RR Lyraes (saturation effects may occur in metal-rich RR Lyraes).

\section{Missclassified and unusual stars}

The following stars were included in the HIPPARCOS list of RR Lyraes but are probably not RR Lyraes:

1. CW Lup. Our spectra show a constant velocity of $31.7 \mathrm{~km} \mathrm{~s}^{-1}$ based on 3 well-placed observations. The HST Guide Star Catalogue shows a star at exactly the HIPPARCOS coordinates and this is the star we observed $\left(\alpha(2000.0)=14^{\mathrm{h}} 20^{\mathrm{m}} 28.2^{\mathrm{s}} ; \delta(2000.0)=\right.$ $\left.-44^{\mathrm{d}} 31^{\mathrm{m}} 56^{\mathrm{s}}\right)$. However, there is another star of similar brightness approximately 1 arcmin away and probably this is the variable.

2. UY Scl. Our spectra show a star of much later spectral type (weak $\mathrm{H} \gamma$, strong molecular absorption bands) with radial velocity variations of $\approx 40$ $\mathrm{km} \mathrm{s}^{-1}$. A search of the HST Guide Star Catalogue at the HIPPARCOS position $\left(\alpha(2000.0)=00^{\mathrm{h}} 14^{\mathrm{m}} 45.7^{\mathrm{s}}\right.$; $\left.\delta(2000.0)=-39^{d} 14^{m} 36^{s}\right)$ shows no other star of 
similar brightness within a 6 arcmin radius. The star is clearly a variable but has been missclassified as an RR Lyrae.

3. IV Pav. The GCVS lists this star as having $\Delta V=0.4$ but no period is given. Our spectra show a variable radial velocity, $18 \leq v r \leq 39 \mathrm{~km} \mathrm{~s}^{-1}$, but the lines are broader and shallower than for other RR Lyraes. Probably the star is a short-period Eclipsing Variable.

4. FW Lup and IK Hya. Both these stars showed relatively small velocity amplitudes. The GCVS also shows they have relatively small light amplitudes (FW Lup $\Delta V=0.40$ and IK Hya $\Delta V=0.46$ ). The stars therefore show the characteristics of the socalled Anomalous Cepheids, namely the periods imply fundamental mode pulsation ( $\mathrm{FW}$ Lup $\mathrm{P}=0.48$ days, IK Hya $\mathrm{P}=0.65$ days) but the light and velocity curves are more compatible with overtone pulsation. For further discussion of Anomalous Cepheids see Teays \& Simon (1985) and for other candidate stars see Paper I.

Acknowledgements. We would like to thank Eduardo Ojero for his help during the observations in Calar Alto and the financial support of NATO through Grant number CRG 940746.

\section{References}

Alonso A., Arribas S., Martínez-Roger C., 1996, A\&AS 117, 227

Barnes T.G., Moffett T.J., Hawley S.L., Slovak M.H., Frueh M.L., 1988, ApJS 67, 403

Bell R.A., Paltoglou G., Tripicco M.J., 1994, MNRAS 268, 771

Blackwell D.E., Lynas-Gray A.E., 1994, A\&A 282, 899

Borges A.C., Idiart, T.P., De Freitas Pacheco J.A., Thevenin F., 1995, AJ 110, 2408

Burki G., Meylan G., 1986, A\&A 156, 131

Butler D., 1975, ApJ 200, 68

Cacciari C., Clementini G., Prevot L., et al., 1987, A\&AS 69 135

Carney B.W., Laird J.B., Latham D.W., Kurucz R.L., 1987, AJ 94, 1066

Cayrel de Strobel G., Hauck B., Francois P., et al., 1992, A\&AS 95, 273

Clementini G., Carreta E., Gratton R., et al., 1995, AJ 110, 2319

Clementini G., Tosi M., Merighi R., 1991, AJ 101, 2168

Clube S.V.M., Evans D.S., Jones D.H.P., 1969, Mem. R. Astron. Soc. 72, 101

Drake J.J., Laming J.M., 1995, Observatory 115, 118

Edvardsson B., Andersen J., Gustafsson B., et al., 1993, A\&AS
102,603

Favata F., Micela G., Sciortino S., 1996, A\&A 311, 951

Fernley J., Barnes T.G., 1997, A\&AS (in press) (Paper I)

Fernley J., Barnes T.G., 1996, A\&A 312, 957

Fernley J., Lynas-Gray A.E., Skillen I., et al., 1989, MNRAS 236, 447 G., 1990, MNRAS 247, 287

Furhmann K., Axer M., Gehren T., 1994, A\&A 285, 585 An algorithm for the integration of unequally spaced data Compt. J., 15, pp. 80-83, 1972

Griffin R., 1979, in: The Observatory. Cambridge (eds.). A photometric atlas of the spectrum of Procyon $\lambda \lambda 3140-7470 \AA$

Hawley S.L., Barnes T.G., 1985, PASP 97551

Hawley S.L., Jeffreys W.H., Barnes T.G., Lai W., 1986, ApJ 302,626

Imbert M., 1980, A\&A 86, 259

Jones R.V., Carney B.W., Latham D.W., 1988, ApJ 326, 312

Joy A.H., 1949, PASP 62, 60

Joy A.H., 1938, PASP 50, 302

Kemper E., 1982, AJ 87, 1395

King J.R., Boesgaard A.M., 1995, AJ 109, 383

Kinman T.D., Caretta E., 1992, PASP 104, 111

Kholopov P.N., Samus N.N., Frolov M.S., et al., 1990, in: General Catalogue of Variable Stars. Ed. Nauka, Moscow

Kurucz R.L., 1979, ApJS 40, 1

Kurucz, R.L., Peytremann, 1975, Smithsonian Atrophys. Obs. Special Report, 362

Lambert D.L., Heath J.R., Lemke M., Drake J., 1996, ApJ 103, 183

Layden A.C., 1994, AJ 108, 1016

Liu T., 1991, PASP 103, 205

Liu T., Janes K.A., 1990, ApJ 354, 273

Lub J., 1979, AJ 84, 383

Meylan G., Burki G., Rufener F., et al., 1986, A\&AS 64, 25

Oke J.B., 1966, ApJ 145, 468

Preston G.W., 1959, ApJ 130, 507

Preston G.W., Spinrad H., Varsavsky C.M., 1961, ApJ 133, 484

Saha A., White R.E., 1990, PASP 102, 148

Smalley B., Dworetsky M.M., 1993, A\&A 271, 515

Smith H.A., 1990, PASP 102, 124

Sokolov N.A., 1995, A\&AS 110, 553

Solano E., Fernley J., 1997, A\&AS 122, 131

Strugnell P., Reid N., Murray C.A., 1986, MNRAS 220, 413

Suntzeff N.B., Kinman T.D., Kraft R.P., 1991, ApJ 367, 528

Teays T.J., Simon N.R., 1985, ApJ 290, 683

Thévenin F., 1989, A\&AS 77, 137

Tomkin J., Woolf V.M., Lambert D.L., Lemke M., 1995, AJ 109,2204

van't Veer-Menneret C., Mégessier C., 1996, A\&A 309, 879

Varsavsky C.M., 1960, ApJ 131, 623

Woolley R., Ali K., 1966, Roy. Obs. Bull. 114 